\title{
Therapieeffekte bei auditiver Verarbeitungs- und Wahrnehmungsstörung im Kindesalter
}

C. Welzel, W. Vorwerk, Ch. Rasinski, U. Sievert, S. Bartel-Friedrich

\section{Einleitung}

Sind Kinder nicht fähig, auditive Reize zu deuten, sie voneinander zu unterscheiden oder mit Bekanntem zu assoziieren, liegt oft eine sog. AVWS (Auditive Verarbeitungs- und Wahrnehmungsstörung) i.S. einer zentralen Teilleistungsschwäche vor. Sie fällt häufig im Vorschul- oder frühen Schulalter durch Einschränkungen der Sprachverständnisfähigkeit im Störschall, des Sprachlautdiskriminationsvermögens oder der Hörmerkspanne auf. Häufig bestehen therapeutisch schwer beeinflussbare Sprachstörungen (z.B. Dyslalie,

Dysgrammatismus).

\section{- spezifische AVWs}

ohne weitere erkennbare Ursach

- unspezifische AVWS

in Kombination mit anderen Störungen oder als Symptom solcher Störungen *

(* Auffnerksamkeitsstörung, Hyperaktivităt, Lernstörungen, Störungen der Speicher- und Abruffunktionen von Gedächtnisfähigkeiten,

Spracherwerbsstörungen, Einschränkungen der allgemeinen Intelligenz)

\section{Diagnostik}

- phoniatrisch / pädaudiologisch

A) Screening (nach Nickisch): Tonschwellenaudiometrie Mottier-Test (HMS)

Hannoverscher Lautdiskriminationstest (HLDT) Sprachaudiometrie im Störschall Regiometrie

B) ausführliche AVWS-Diagnostik (bei pathol. Screening) - subjektive audiologische Tests

Kindersprachoudiometrie (Sprachtest n. Jakohi (Albred ) dichotischer Diskriminationstest nach Uttenweiler

Hörtest mit zeitkomprimierter Sprache

Hörmerkspanne (Zahlen, Sätze)

Carhart-Test (Hörermüdung)

- objektive audiologische Tests

Tympanometrie

Stapediusreflexe

Otoakustische Emissionen (TEOAE, DPOAE)

evozierte Potentiale (BERA, CERA)

- logopadische Diagnostik

Sprech- und Sprachstatus, MVPT, CMM, DRT

- interdisziplinär

psychiatrisch / psychologisch

entwicklungsneurologisch

pädagogisch

\section{Therapie-Optionen}

1. Interventionen zur Verbesserung auditiver

Verarbeitungs- und Wahrnehmungsstörungen

$\longrightarrow$ logopädisches Hörtraining

2. Verfahren zur verbesserten Kompensation gestörter Funktionen

$\longrightarrow$ psychologisch / pädagogisch

3. Kompensatorische Verfahren zur Verbesserung der akustischen Signalqualität

- Hörgeräteversorgung

Indikation: - auffällige Befunde in $>2$ der subjektiven bzw. objektiven audiologischen Tests - pathologisches Sprachverständnis im

Störgeräusch mit einem

Diskriminationsverlust von mehr als $30 \%$

\section{Material und Methoden}

Untersuchungsgruppe:

Alter bei Erstdiagnostik:

Alter bei Kontrolle:

$\varnothing 8$ Jahre, 7 Monate

$\varnothing 9$ Jahre, 10 Monate

Zeitpunkt der Erstdiagnostik: $\quad 11 / 2000-03 / 2004$

Ergebnisse der Erstdiagnostik:

$$
\begin{array}{ll}
-13 \text { spezifische AVWS }\left(\sigma^{*}, \rho^{7}\right) & 39 \% \\
-20 \text { unspezifische AVWS }\left(\sigma^{11}, p^{9}\right) & 61 \%
\end{array}
$$

Kontrolluntersuchung:

$06 / 2002-03 / 2005$

Kontrollzeitpunkt: $\varnothing 14$ Monate nach Erstdiagnostik ( 7 - 28 Monate)

Die Kontrolluntersuchung umfasste ausschliesslich die bei der Erstdiagnostik pathologischen audiologischen und logopädischen Befunde.

Es wurden die zur Erstuntersuchung identischen

Testverfahren in einer vergleichbaren Testsituation angewendet.

\section{Therapie}

Es wurde nach erfolgter AVWS-Diagnostik allen Kindern ein logopädisches Hörtraining empfohlen.

Bei gleichzeitig bestehenden erheblichen Defiziten im

Bereich der Sprachverständnisfähigkeit im Störschall

(Diskriminationsverlust $>30 \%$ ) erfolgte zusätzlich zur

logopädischen Therapie eine einseitige Hörgeräte-

versorgung.

Kinder mit Begleitstörungen (ADHS, Intelligenzdefizit,

Verhaltensstörungen, LRS etc.) erhielten z.T. eine

weiterführende störungsspezifische Förderung

(psychologisch, neurologisch, pädagogisch,

ergotherapeutisch)

\begin{tabular}{|l|c|c|}
\hline \multicolumn{1}{|c|}{ Therapieart } & n & n (\%) \\
\hline $\begin{array}{l}\text { nur logopädisches } \\
\text { Hörtraining }\end{array}$ & 6 & $18 \%$ \\
\hline $\begin{array}{l}\text { logopädisches Hörtraining }+ \\
\text { andere Therapieformen }\end{array}$ & 23 & $70 \%$ \\
\hline nur andere Therapieformen & 4 & $12 \%$ \\
\hline Hörgeräteversorgung & 7 & \\
\hline
\end{tabular}

mittlere Therapiedauer (Hörtraining):

$=30$ Therapieeinheiten a 45 Minuten

\section{Ergebnisse}

(A) Häufigste prätherapeutische Defizite

$\mathbf{n}=\mathbf{3 3}$

- Sprachverständnis im Störschall

$$
48 \% \text { (16) }
$$

- dichotische Diskrimination

$$
91 \%(30)
$$

- Hörmerkspanne (HMS)

$$
\text { Zahlen: } \quad 82 \%(30)
$$

Sätze: $\quad 64 \%(21)$

Mottier: $94 \%(31)$

- Lautdiskrimination (HLDT)

$$
67 \%(22)
$$

- Hörtest mit zeitkomprimierter Sprache $94 \%(31)$

- Dysgrammatismus

$$
48 \%(16)
$$

(B) modalitätsspezifische Therapieeinflüsse

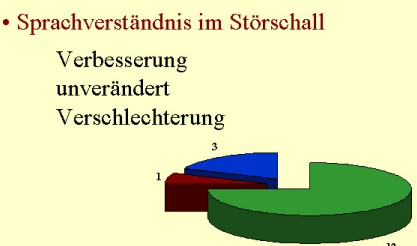

$\mathrm{n}=\mathbf{1 6}$

$75 \%(12)$

$6 \% \quad(1)$

$19 \% \quad(3)$

- dichotische Diskrimination

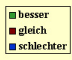

Verbesserung

davon Normalisierung

unverändert

$7 \% \quad(2)$

Als Verbesserung wurde anch die Umwandlung eines intitial monauralen in ein binaurales Wortverständris (auch pathologisch) gewertet.
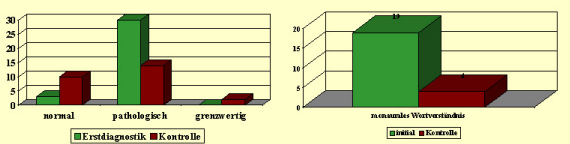

• Hörmerkspanne (HMS)

Mottier:

Verbesserung

davon Normalisierung

erändert

1 Kind konnten nicht nachuntersucht werden.

- Lautdiskrimination (HLDT)

$$
\begin{aligned}
& \text { Verbesserung } \\
& \text { davon Normalisierung }
\end{aligned}
$$

erändert

Kinder konnten nicht nachuntersucht werden.

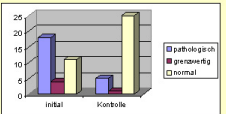

- Hörtest mit zeitkomprimierter Sprache

$$
\begin{aligned}
& \text { Verbesserung } \\
& \text { davon Normalisierung }
\end{aligned}
$$

unverändert

3 Kinder konnten nicht nachuntersucht werden.

- Dysgrammatismus

$$
\text { Verbesserung }
$$$$
\mathrm{n}=16
$$

unverändert

\section{(C) Hörgeräteeffekte}

$$
\mathbf{n}=7
$$

- Sprachverständnis im Störschall

Normalisierung mit und ohne Hörgerät

- dichotische Diskrimination

Verbesserung bei 5 Kindern ( $2 x$ Normalisierung) unverändert bei 2 Kindern

- Lautdiskrimination

Normalisierung bei 5 Kindern, unverändert $2 \mathrm{x}$

- Zeitkompression und Hörmerkspanne

Verbesserung aber keine Normalisierung

- Verbesserung der Sprachkompetenz bei allen Kindern

- bei 2 Kindern ergab sich bei der Kontrolluntersuchung kein Anhalt mehr für das Vorliegen einer AVWS

\section{Schlussfolgerung}

Bei einer auditiven Verarbeitungs- und Wahrnehmungsstörung können durch ein störungsspezifisches logopädisches Hörtraining Verbesserungen in allen untersuchten Modalitäten erreicht werden. Als am wenigsten beeinflussbar zeigten sich das Hörvermögen zeitkomprimierter Sprache, die Hörmerkspanne (insbesondere Mottier) und der
Dysgrammatismus. Durch eine Hörgeräteversorgung können Kinder mit einem ausgeprägten Diskriminationsverlust im Störschall-Sprachaudiogramm erheblich profitieren. Eine Schädigung des peripheren Hörvermögens wurde in keinem Fall festgestellt. 